\title{
Supporting Information \\ Unexpected Solute Occupancy and Anisotropic Polarizability in Lewis Basic Solutions
}

\author{
Siyan $\mathrm{Gao}^{1}$, Yongli Huang ${ }^{1, *}, \mathrm{Xi}$ Zhang ${ }^{2, *}$, Chang Q Sun ${ }^{3}$
}

\section{Structure optimization}

We adopted three possible $\mathrm{Y}^{+}$positions, central-tetrahedron position, above-triangle position, and in-triangle position, as shown in Figure S1. The central-tetrahedron position means that $\mathrm{Y}^{+}$ion is located at the center of a water tetrahedron unit. The $\mathrm{Y}^{+}$ ion has six nearest water neighbors and the distance is the lattice constant $a$. The above-triangle position means that $\mathrm{Y}^{+}$ion is located above three oxygen atoms forming a triangle in a plane. The distance between $\mathrm{Y}^{+}$ion and oxygen is smaller than a. The in-triangle position means that $\mathrm{Y}^{+}$ion is located in the center of oxygen atoms-triangle in a plane. $\mathrm{Y}^{+}$ion has three nearest neighbors and the distance is the smallest. It can be predicted the $\mathrm{Y}^{+}$ion will induce the larger lattice distortion and instability when it get closer to water molecule. We performed structural optimizations of these aqueous solution models. As predicted, the in-triangle position is the most unstable, and the above-triangle position the second. The total energy for central-tetrahedron position, above-triangle position, and in-triangle position are $-133395.04 \mathrm{eV},-133392.11 \mathrm{eV}$, and $-133388.85 \mathrm{eV}$, respectively. The $\mathrm{OH}^{-}$ion was

\footnotetext{
1 School of Materials Science and Engineering, Xiangtan University, Hunan 411105, China (siyangaoxtu@163.com; huangyongli@xtu.edu.cn)

${ }^{2}$ Institute of Nanosurface Science and Engineering, Shenzhen University, Shenzhen 518060, China; (zh0005xi@szu.edu.cn)

${ }^{3}$ NOVITAS, School of Electrical and Electronic Engineering, Nanyang Technological University, Singapore 639798, Singapore; (ecqsun@ntu.edu.sg)
} 
not induced additionally but just changed from a water molecule in the crystal structure.
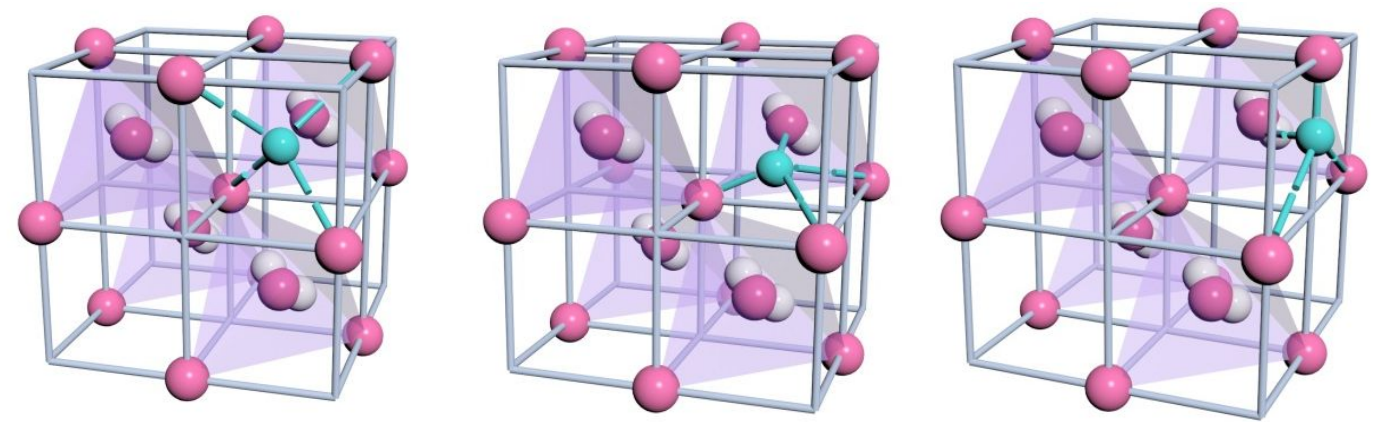

Figure S1. Three different positions of $\mathrm{Y}^{+}$ion. (a) Central-tetrahedron position; (b) Above-triangle position; (c) In-triangle position.

\section{Vibrational mode}

The vibration mode shift positions are confirmed through the same frequency vibrational mode in the frequency ranges of $\omega_{\mathrm{L}}<400 \mathrm{~cm}^{-1}$ and $\omega_{\mathrm{H}}>3000 \mathrm{~cm}^{-1}$. Figure S2 shows specific vibrational modes of hydrogen bond segments, O:H nonbond and $\mathrm{H}-\mathrm{O}$ bond, at different frequencies. The frequency of $253 \mathrm{~cm}^{-1}$ shows the O:H stretching mode and the frequency of $832 \mathrm{~cm}^{-1}$ is the $\mathrm{O}: \mathrm{H}$ bending mode. For the higher frequency, the frequencies of $3087 \mathrm{~cm}-1$ and $3294 \mathrm{~cm}-1$ are H-O symmetric and asymmetric stretching modes, respectively. 
a

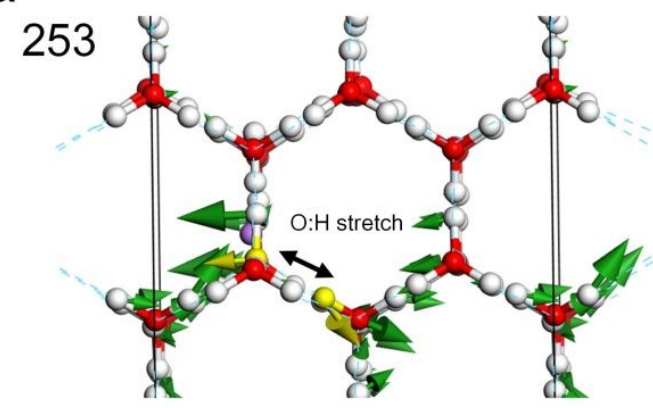

C

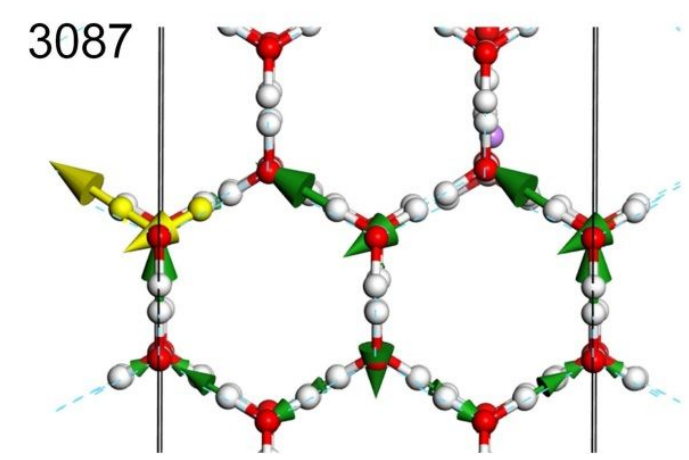

b

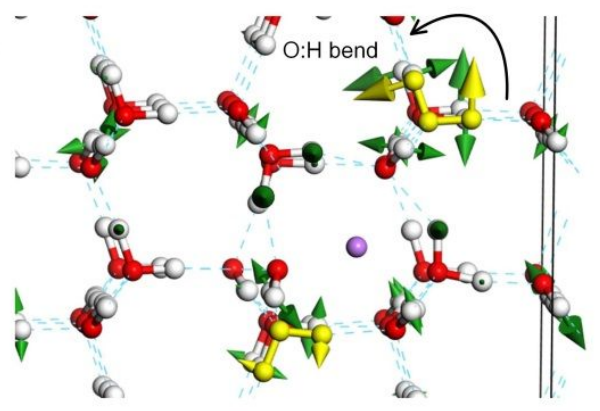

d

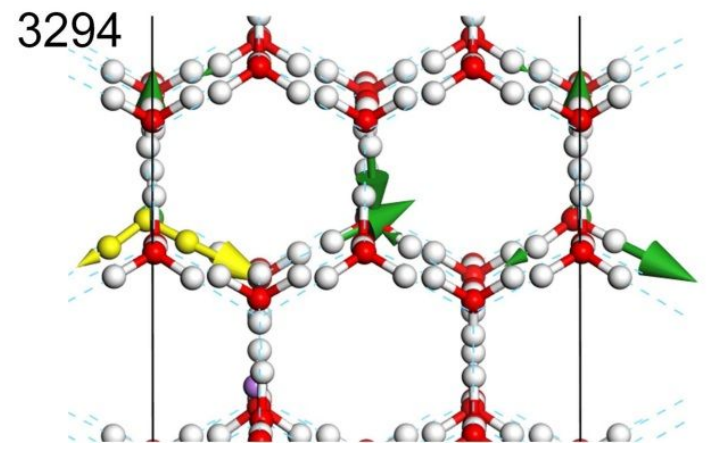

Figure S2. (a) The stretching mode of O:H at $253 \mathrm{~cm}^{-1}$. (b) The bending mode at 832 $\mathrm{cm}^{-1}$. (c) The stretching mode of $\mathrm{H}-\mathrm{O}$ at $3087 \mathrm{~cm}^{-1}$ (symmetric stretching) and 3294 $\mathrm{cm}^{-1}$ (asymmetric stretching), respectively.

\section{MD calculation}

We further employed molecular dynamics (MD) calculation to compute the power spectrum of water containing $\mathrm{OH}^{-}$(supporting information). We carried out $\mathrm{MD}$ calculations using Forcite's package with ab initio optimized forcefield CompassII ${ }^{1}$. The unit cell consisted of 64 water molecules and an $\mathrm{OH}$ - hydroxide. The electronic structure was dynamically relaxed during 10 ps (20000 steps) in the NVT ensemble. The temperature was set as $298 \mathrm{~K}$ and the thermostat was applied by Mass GGM method. The equilibrium structures were gained after $10 \mathrm{ps}$ at the time step of $0.5 \mathrm{fs}$. Further, we computed the power spectra through the Fourier transformation of the velocity autocorrelation function ${ }^{2}, \operatorname{Cor}(\mathrm{t}), \quad I(\omega)=2 \int_{0}^{\infty} \operatorname{Cor}(v(t)) \cos (\omega t) d t$ employing velocity data of all atoms in the dynamic system. 
Figure S3 shows the MD-derived power spectrum of pure water and $\mathrm{OH}^{-}$solution. Figure S4 compares the differential spectra $\left(\mathrm{OH}^{-}\right.$solution subtracted by water). Compared to DFT-derived vibration spectra, the MD-derived power spectrum has the same frequency shifts of $\mathrm{O}-\mathrm{H}$ and $\mathrm{O}: \mathrm{H}$ bonds. The fluctuation was caused by the temperature of $298 \mathrm{~K}$ applied in MD. The results reveal that the $\mathrm{O}: \Leftrightarrow: \mathrm{O}$ repulsion lengthen the opposing $\mathrm{H}-\mathrm{O}$ bond but shorten the subsequent $\mathrm{O}: \mathrm{H}$ cooperatively.

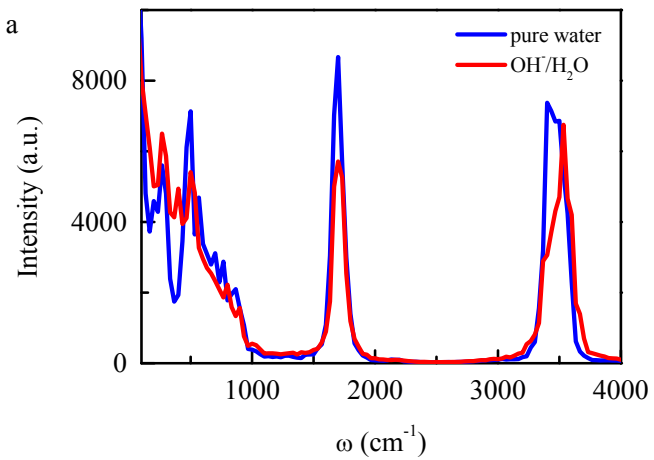

Figure S3. The MD-derived power spectrum of pure water and $\mathrm{OH}^{-}$solution.

a
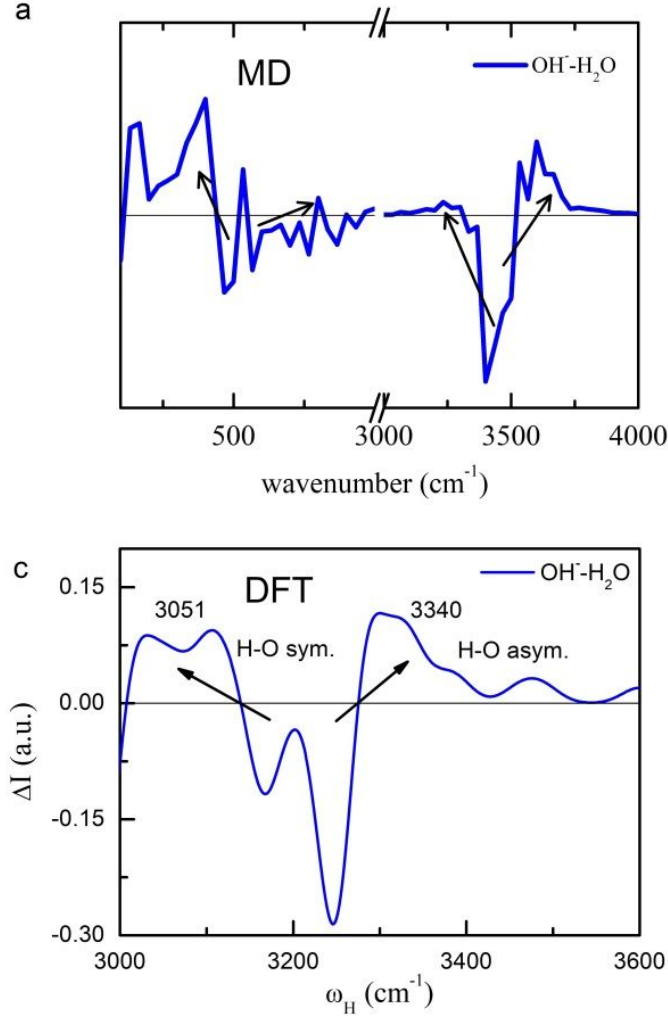

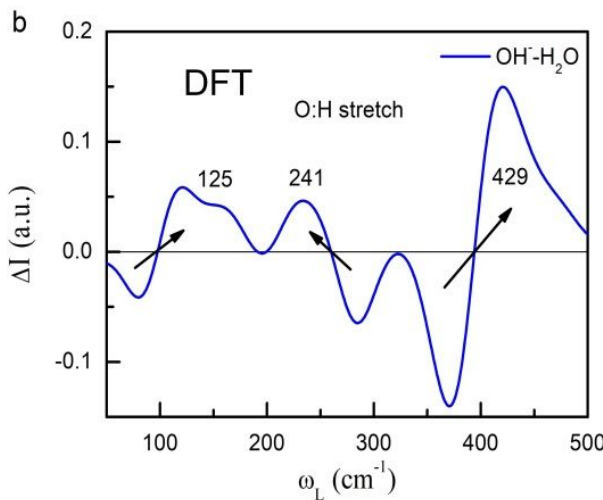

d

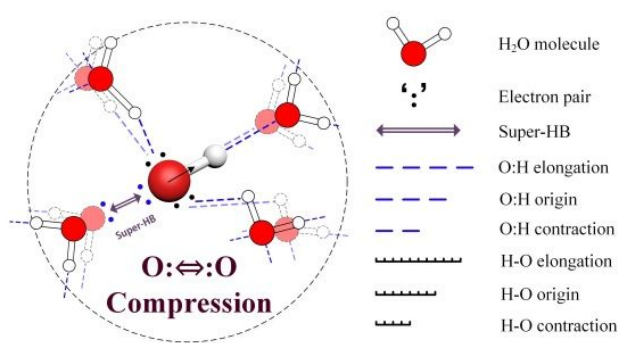

Figure S4. The differential spectra $\left(\mathrm{OH}^{-}\right.$solution subtracted by water) of (a) 
MD-derived power spectrum and DFT-derived vibration spectra for (b) $\omega_{\mathrm{L}}(50 \sim 600$ $\left.\mathrm{cm}^{-1}\right)$ and $(\mathrm{c}) \omega_{\mathrm{H}}\left(3100 \sim 3600 \mathrm{~cm}^{-1}\right)$ between the $\mathrm{OH}^{-} / \mathrm{H}_{2} \mathrm{O}$ solutions and pure water. (d) The diagram of the first hydration shell of $\mathrm{OH}^{-}$-contained water. 


\section{References}

1. Sun, H.; Jin, Z.; Yang, C.; Akkermans, R. L.; Robertson, S. H.; Spenley, N. A.; Miller, S.; Todd, S. M., COMPASS II: extended coverage for polymer and drug-like molecule databases. Journal of molecular modeling 2016, 22 (2), 47.

2. (a) Kang, D.; Dai, J.; Hou, Y.; Yuan, J., Structure and vibrational spectra of small water clusters from first principles simulations. The Journal of Chemical Physics 2010, 133 (1), 014302;

(b) Sun, C. Q.; Zhang, X.; Zheng, W., The hidden force opposing ice compression. Chemical Science 2012, 3 (5), 1455-1460. 